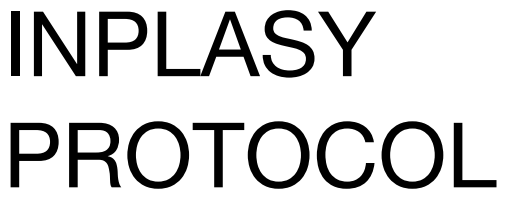

To cite: Wang et al. The efficiency of

Hydroxychloroquine for the treatment of primary Sjögren's Syndrome: a systematic review and meta-analysis. Inplasy protocol 202090063. doi: 10.37766/inplasy2020.9.0063

Received: 15 September 2020

Published: 15 September 2020

Corresponding author: Jianping Tang

1910829@tongji.edu.cn

Author Affiliation:

Tongji Hospital, Tongji

University School of Medicine

Support: NSFC.

Review Stage at time of this submission: Preliminary searches.

Conflicts of interest:

The author declared no conflicts of interest.

\section{The efficiency of Hydroxychloroquine for the treatment of primary Sjögren's Syndrome: a systematic review and} meta-analysis

Wang, X1; Zhang, T2; Guo, Z3; Pu, J4; Farooq, R5; Feng, R6; Fang, $\mathrm{X}^{7}$; Song, J8; Liang, Y9; Wu, Z10; Pan, S11; Zhang, M12; Tang, J13.

Review question / Objective: P: primary Sjögren's Syndrome patients; I: hydroxychloroquine; C: hydroxychloroquine/nonhydroxychloroquine; O: Schirmer's test, uSFR, CRP, ESR, and immunoglobulins (IgG, IgM, and $\lg A)$; $S$ : randomized controlled clinical trials (RCTs).

Condition being studied: Primary Sjögren's Syndrome (pSS), with an estimated worldwide prevalence of $0.06 \%$ (1), is a chronic and systemic autoimmune disease that is characterized by the focal lymphocytic infiltration of the exocrine glands causing the oral and ocular dryness, fatigue, and pain. These three symptoms are present in more than $80 \%$ of the pSS patients and greatly compromise their quality of life (2). In addition to the clinical manifestations of salivary and lacrimal glands, a subset of patients also showed extraglandular involvement with the development of signs and symptoms in other organs including skin, joints, lungs, gastrointestinal tract, kidneys, nervous system, and circulatory system. These systemic complications occur in approximately $30-40 \%$ of the pSS patients. The diagnosis of pSS is often made based on oral and ocular dryness, fatigue, and pain. Oral and ocular dryness-based diagnosis is made by measuring the unstimulated salivary flow rate(uSFR), and Schirmer's test. However, sometimes systemic complications provide preliminary clues to diagnose the pSS. Moreover, antiSSA antibodies, often associated with anti-SSB antibodies, should be assessed in case of suspected patients.

INPLASY registration number: This protocol was registered with the International Platform of Registered Systematic Review and Meta-Analysis Protocols (INPLASY) on 15 September 2020 and was last updated on 15 September 2020 (registration number INPLASY202090063).

\section{INTRODUCTION}

Review question / Objective: P: primary Sjögren's Syndrome patients; I: hydroxychloroquine; C: hydroxychloroquine /non-hydroxychloroquine; 0 : Schirmer's t e st, u S F R, CRP, ESR, a nd immunoglobulins (IgG, IgM, and IgA); S: randomized controlled clinical trials (RCTs).

Condition being studied: Primary Sjögren's Syndrome ( $p S S)$, with an estimated 
worldwide prevalence of $0.06 \%$ (1), is a chronic and systemic autoimmune disease that is characterized by the focal lymphocytic infiltration of the exocrine glands causing the oral and ocular dryness, fatigue, and pain. These three symptoms are present in more than $80 \%$ of the pSS patients and greatly compromise their quality of life (2). In addition to the clinical manifestations of salivary and lacrimal glands, a subset of patients also showed extra-glandular involvement with the development of signs and symptoms in other organs including skin, joints, lungs, gastrointestinal tract, kidneys, nervous system, and circulatory system. These systemic complications occur in approximately $30-40 \%$ of the pSS patients. The diagnosis of pSS is often made based on oral and ocular dryness, fatigue, and pain. Oral and ocular dryness-based diagnosis is made by measuring the unstimulated salivary flow rate(uSFR), and Schirmer's test. However, sometimes systemic complications provide preliminary clues to diagnose the pSS. Moreover, antiSSA antibodies, often associated with antiSSB antibodies, should be assessed in case of suspected patients.

\section{METHODS}

Participant or population: primary Sjögren's Syndrome patients.

Intervention: Hydroxychloroquine.

Comparator: Hydroxychloroquine/nonhydroxychloroquine.

Study designs to be included: Randomized controlled clinical trials (RCTs).

Eligibility criteria: This study was performed following the preferred reporting items for systematic reviews and metaanalyses (PRISMA) guidelines.

Information sources: PUBMED, EMBASE, CENTRAL, LILACS, Clinical Trials databases, and master and doctor thesis database.
Main outcome(s): Schirmer's test, uSFR, CRP, ESR, and immunoglobulins (IgG, IgM, and $\lg A)$.

Quality assessment / Risk of bias analysis: The quality of the studies was assessed using the Downs and Black quality assessment tool that contains a list of 27 criteria for evaluation of the reporting, external validity, internal validity-bias, confounding (selection bias), and the power of assessed studies. The level of evidence represented by each study was categorized based on the Oxford Centre for Evidence-Based Medicine Levels of Evidence (OCEBM. http://www.cebm.net/ index.aspx $?=5653)$. The OCEBM classifies the evidence levels of the research into five grades, with levels ranging from level 1 to level 5.

Strategy of data synthesis: Forest plots.

Subgroup analysis: Intervention duration and combind treatment.

Sensibility analysis: We performed sensitivity analysis by removing the lowquality studies and then compared the differences between the results.

Country(ies) involved: China.

Keywords: hydroxychloroquine; Sjögren's Syndrome; systematic review; metaanalysis.

Contributions of each author:

Author 1 - Xuan Wang.

Author 2 - Tongyangzi Zhang.

Author 3 - Zizhen Guo.

Author 4 - Jincheng Pu.

Author 5 - Farooq Riaz.

Author 6 - Run Feng.

Author 7 - Xingxing Fang.

Author 8 - Jiamin Song.

Author 9 - Yuanyuan Liang.

Author 10 - Zhenzhen Wu.

Author 11 - Shengnan Pan.

Author 12 - Min Zhang.

Author 13 - Jianping Tang. 